\title{
Half-Chain Trastuzumab Nanoconjugates Enhance Antitumor Activity in HER2+ breast cancer
}

\author{
Marta Truffi $^{1}$, Miriam Colombo², Luca Sorrentino' ${ }^{1}$, Serena Mazzucchelli ${ }^{1}$, Laura Pandolfi ${ }^{2}$, Arianna \\ Bonizzi $^{1}$, Davide Prosperi ${ }^{2}$, Fabio Corsi ${ }^{1}$ \\ ${ }^{1}$ Department of Biomedical and Clinical Sciences "L. Sacco", University of Milan \\ via G. B. Grassi, 74, Milan, Italy \\ marta.truffi@unimi.it; luca.sorrentino1@unimi.it; serena.mazzucchelli@unimi.it; arianna.bonizzi@unimi.it; \\ fabio.corsi@unimi.it \\ ${ }^{2}$ Department of Biotechnologies and Biosciences, University of Milan-Bicocca \\ piazza della Scienza 2, Milan, Italy \\ miriam.colombo@unimib.it; pandolfi.la@gmail.com; davide.prosperi@unimib.it
}

\section{Extended Abstract}

The human epidermal growth factor receptor 2 (HER2) is overexpressed in about 20-30\% of all breast cancers, where it triggers multiple downstream pathways required for the abnormal proliferation of cancer cells.[1] In HER2+ breast cancer population, anti-HER2-specific therapies have revolutionized the clinical management of the disease. In particular, since its approval by the Food and Drug Administration, the monoclonal antibody trastuzumab (TZ) has represented the gold standard for the treatment, with improved disease-free and overall survival.[2] In vivo, the antiproliferative mechanism of $\mathrm{TZ}$ is prominently hold by recruitment of antitumor immunity, through a mechanism called antibody-dependent cell-mediated cytotoxicity (ADCC). However, TZ may also exert direct blockade of HER2-mediated downstream signaling, with inhibition of pathways supporting tumor growth and proliferation. $[3,4]$ Several nanoconjugates have exploited TZ as a potent and effective targeting molecule to improve specificity for HER2-overexpressing tumor cells.[5-7] However, there is no real investigation on how the activity and the therapeutic efficacy of TZ may vary due to conjugation and spatial exposure on the surface of nanoparticles.

Small colloidal iron oxide nanoparticles were functionalized with multiple half chains of TZ upon reduction of disulfide bridges between the two heavy chains of the IgG. Resulting nanoparticles (MNP-HC) showed hydrodynamic diameter of $48.7 \pm 1.0 \mathrm{~nm}$ and $\zeta$-potential of $-44.5 \pm 9.9 \mathrm{mV}$. MNP-HC were assessed for their capability to interact with human breast cancer cell lines and compared to aspecific IgG-coupled nanoparticles or same dosage of free TZ. MNP-HC revealed dosedependent binding to breast cancer cells, with increasing percentage of positivity for those cell lines overexpressing HER2. Specificity of MNP-HC for HER2 was confirmed by competition assay in presence of a molar excess of free TZ. Interaction of MNP-HC with TZ-sensitive cells induced tyrosine-specific phosphorylation in the catalytic site of HER2 receptor even at low dosage, and rapid cellular uptake by endocytosis. Treatment with MNP-HC decreased viability of breast cancer cells, leading to enhanced antitumor efficacy as compared to equal dosage of free TZ. Reduced viability was associated with increased expression of the cell cycle inhibitor p27Kip1 and cell cycle arrest in G1 phase. MNP-HC did not loose capability to activate ADCC, further indicating valuable potential for the nanocomplex. Moreover, MNP-HC were tested on TZresistant breast cancer cells, where they were able to induce direct reduction of cell viability and sensitization to chemotherapy.

In conclusion, multiple and oriented immobilization of $\mathrm{TZ}$ half chains on carriers with narrow size amplified recognition of HER2 and enhanced anti-HER2 efficacy of TZ antibody. Improved antitumor performance of MNP-HC was mainly attributed to sustained blockade of HER2-mediated downstream signaling, although capability to prime ADCC was still maintained. Powerful inhibition of HER2 signaling also promoted responsiveness of TZ-resistant cells, thus suggesting MNP-HC as strategy option for drug re-sensitization in the treatment of HER2-positive breast cancer. 


\section{References}

[1] S. Loibl and L. Gianni, "HER2-positive breast cancer," Lancet Lond. Engl., vol. 389, no. 10087, pp. $2415-2429,2017$.

[2] D. Slamon et al., "Adjuvant trastuzumab in HER2-positive breast cancer," N. Engl. J. Med., vol. 365, no. 14, pp. 12731283, 2011.

[3] J. Baselga, J. Albanell, M. A. Molina, and J. Arribas, "Mechanism of action of trastuzumab and scientific update," Semin. Oncol., vol. 28, no. 5, Suppl 16, pp. 4-11, 2001.

[4] L. Arnould et al., "Trastuzumab-based treatment of HER2-positive breast cancer: an antibody-dependent cellular cytotoxicity mechanism," Br. J. Cancer, vol. 94, no. 2, pp. 259-267, 2006.

[5] S. J. Lee et al., "Functionalized Magnetic PLGA Nanospheres for Targeting and Bioimaging of Breast Cancer," $J$. Nanosci. Nanotechnol., vol. 18, no. 3, pp. 1542-1547, 2018.

[6] Z. Cai et al., "Local Radiation Treatment of HER2-Positive Breast Cancer Using Trastuzumab-Modified Gold Nanoparticles Labeled with 177Lu," Pharm. Res., vol. 34, no. 3, pp. 579-590, 2017.

[7] J. K. Noh et al., "Herceptin-functionalized pure paclitaxel nanocrystals for enhanced delivery to HER2-postive breast cancer cells," Int. J. Pharm., vol. 513, no. 1-2, pp. 54-553, 2016. 